\title{
Importance of magnetic resonance imaging in the diagnosis of breast hamartoma
}

\author{
Altunkeser $\mathrm{A}^{1}$, Arslan $\mathrm{FZ}^{1 *}$, Eryilmaz $\mathrm{MA}^{2}$ and Oner $\mathrm{O}^{1}$ \\ ${ }^{1}$ Department of Radiology, University of Health Science, Konya Training and Research Hospital, Konya, Turkey \\ ${ }^{2}$ Department of General Surgery, University of Health Science, Konya Trainning and Research Hospital, Konya, Turkey
}

\begin{abstract}
Aim: Hamartomas are benign breast lesions. Radiologic and clinical evaluation has great importance in the diagnosis for reducing unnecessary procedures. We intend to investigate the contribution of magnetic resonance imaging (MRI) in the diagnosis of hamartomas

Method: Our research has been conducted retsospectively, a total of 55 breast hamartomas were reassessed using mammography (MG) and MRI. In the detection of morphological features of hamartomas were compared efficacy of MG and MRI. ADC values were obtained corresponding to lesion localization and normal breast parenchyma.
\end{abstract}

Result:MRI was significantly superior to MG in detecting pesudo-capsule and size $(\mathrm{p}<0,001)$. There was no significant difference between enhancement pattern and ADC values obtained from breast tissue and hamartoma.

Conclusion: Conclusionally, we assume that MRI can provide more detailed information in difficult cases which have not classical mammographic appearances, so MRI can be considered as an alternative imaging for accurate diagnosis and prevent unnecessary biopsies and surgeries.

\section{Introduction}

Hamartomas are relatively rare, well-circumscribed, slow-growing lesions of breast comprised of glandulary and stromal components and those may closely resemble that of normal breast tissue even fibrocystic foci can be seen within them similar to normal breast tissue. This make it difficult or impossible to diagnose pathologically. Since the pathological appearance is similar to that of normal breast tissue on Fine Needle Aspiration Biopsy, increasing the radiological diagnostic accuracy by defining these benign lesions features is of great importance in establishing a true picture of the actual incidence of hamartomas [1]. They are not surrounded by a real capsule and are separated from adjacent breast parenchyma by a pseudocapsule. The incidence of hamartomas is approximately $4.8 \%$ overall benign breast lesions but is getting increasingly more frequent due to breast cancer screenning programmes. It generally seen in middle-aged women [2]. On mammography (MG), hypo-hyperechoic breast-in breast appearance can be seen depending on the predominance of fibroglandular or fat tissue contained. Generally, the internal structure is heterogeneous in appearance and contains a large number of hypo and hyperechogenic areas on ultrasonography (US) [3]. On Magnetic resonance imaging (MRI), hamartomas generally seen as well-circumcribed mass within heterogenous structure, showing varying degree of intensity on T1and T2-weighted imaging (T1W,T2W) depending on proportion of fibroglandulary tissue [4].

Mammographic and ultrasonographic features of hamartomas are well known, but MRI images are less known [5]. Especially in breasts with dense parenchima patterns, fibroglandular tissue and mammographic appearance of lesion is superposing, so it is almost impossible to distinguish lesion borders and to measure their size in certain cases in which the pseudo-capsule is not apparent. On present study, we elucidate the contribution of the diagnosis of hamartoma by examining the features of them on unenhanced, contrastenhanced dynamic and diffusion-weighted (DW) MRI in addition to mammography. We intend to investigate the contribution of MRI in the diagnosis of hamartomas, either alone or integrated into MG and also explored the role of MRI in reducing unnecessary biopsies and unnecessary surgeries regarding the importance of radiologic and clinical evaluation of Hamartomas.

\section{Material and method}

On our research, 46 patients diagnosed with hamartoma and a total of 55 breast hamartomas have been retrospectively reassessed using MG and MRI during a 7-years-old period from 2010 to 2017 . Ethical approval obtained from a local commitee of Health Science Univercity of Konya Trainning and Research center, according to Helsinki Declaration. MG and MRI of all subsequents were assessed by only a single radiologist with15-year experience in the field of breast imaging. Breast density has been mammographically categorized into four groups according to the 5th edition ofAmerican College of Radiology BI- RADS [6]. We defined type A and B breast pattern as dominantly lipomatous type 1, dominantly fibroglandulary type 2 was also included type $\mathrm{C}$ and $\mathrm{D}$

*Correspondence to: Arslan FZ, Konya Training and Research Hospital, Department of Radiology, Haci Saban Mah, Meram New Road Avenue, Meram, Konya, Turkey, Tel: 05064382430; Fax: 03323236723; E-mail: Zeynep_a1002@hotmail.com

Key words: breast hamartoma, magnetic resonance imaging, mammography, apparent diffusion coeficiency

Received: May 17, 2018; Accepted: May 25, 2018; Published: May 30, 2018 
breast pattern. MG examinations were performed using a Full Field Digital Mammography System (mammomat inspiration, Siemens, Erlangen, Germany). Mammographically, the size of the hamartoma examined, presence of the pseudo-capsule and calcification was evaluated, the predominancy of fibroglandular or fat tissue contained were also noted. All examinations were performed using dedicated 16-channel double breast coil with 1.5 Tesla MRI (Magnetom Aera; Siemens Healthcare, Erlangen, Germany) equipped with 45-mT / m gradients and the patient is placed in the prone position on the table. The image protocol was bilateral, coronal flashed-grappa (TR / TE: 417/11 ms, matrix: 352x384, slice thickness: 3mm, FOV: 180-500mm) 352x384, slice thickness: 3mm, FOV: $280-300 \mathrm{~mm}$ ) T1W started with sequences. Then T2W TIRM (TR / TE: 2770/66 ms, matrix: 352x384, inversion time: $150 \mathrm{~ms}$, flip angle: 150 degrees, spatial resolution: $0.7 \mathrm{x}$ $0.7 \times 2 \mathrm{~mm}$, acquisition time: 3 minutes 26 seconds) DWI (TR / TE: $6200 / 88$, long distance resolution: $2.7 \times 2.7 \times 4 \mathrm{~mm}$, slice thickness: $3 \mathrm{~mm}$, FOV: $258-300 \mathrm{~mm}, \mathrm{~B}$ values 0 and $800 \mathrm{~s} / \mathrm{mm} 2$, spectral fat saturation and acquisition time: Minute 47 seconds). Dynamic study was obtained with FLASH (TR / TE: 4.79 / $1.70 \mathrm{~ms}$, spatial resolution: $0.8 \times 0.8 \times 1.3$ $\mathrm{mm}$, cross-sectional thickness: $1.6 \mathrm{~mm}$, FOV: $318-500 \mathrm{~mm}$ ). Finally, assessment of the DWI and apparent diffusion coefficient (ADC) map yielded ADC values corresponding to lesion localization and normal breast parenchyma via region of interest (ROI). The comprasion was performed using the Mann-Whitney test. The efficacy of MRI and MG in the detection of hamartoma size and pseudocapsule was investigated. FisherExact, Sign Test and Man-Whitney U test was used to compare variables.

\section{Result}

The age of all reported individuals varied between 34 and 73 yearsold mean age was 52 and all of the participants were female. There were 55 hamartom in 46 patients, 28 of which were in the right breast and 27 in the left breast. Type 1 parenchyma pattern was observed in 13 patients (26\%), while type 2 parenchyma pattern was observed in 37 patients (74\%). 42 patients were evaluated with MG, 28 patients were assessed with MRI and while the 25 patients were examined using both MRI and MG. The mean diameter of the hamartomas on MRI was $5 \mathrm{~cm}$, and it was $3 \mathrm{~cm}$ on $\mathrm{MG}(\mathrm{p}=0,006)$. Hamartoma pseudocapsule was noted in 27 patients and not noted in 1 patient on MRI. On MG, while 10 of which were noticed pseudo-capsule, 17 of them were unencapsulated $(p<0,001)$ (Table 1) (Figure 1a,b). In 30 of 32 hamartomas; early enhancement was not seen on dynamic MRI, only 2 of these were demonsrated early enhancement. All patients except 1 patient showed type 1 contrast enhancement pattern, type 2 contrast enhancement pattern was observed in 1 patient.

Diffusion restriction was not observed in all patients except 2 cases on DWI. Type 1 contrast pattern without early contrast enhancement was observed in 2 cases demonstrating diffusion restriction. There was no significant difference between ADC values obtained from breast tissue and hamartoma $(\mathrm{p}=0.009)$ (Table 2). Biopsy results were obtained in 12 of 55 hamartomas included in our the study and resulted as breast tissue. The rest of these were diagnosed radiologically and clinically as hamartomas.

\section{Discussion}

Hamartomas are usually located in the upper outer quadrant of the breast and are generally in the form of soft mobile masses, with these physical examination findings hamartomas can easily be mistaken with other benign lesions such as fibroadenomas [7]. The insufficiency of the pathologic diagnosis increases the importance of radiological imaging methods, especially in cases who do not have the typical appearance of hamartomas. Because hamartomas contain fibroglandular and fatty tissue in a wide variety of proportions, this leads many appearances to be seen on the MG and USG. On MG, hamartomas frequently seen as well circumscribed heterogenous solid mass lesions composed of different amount of fat or fibroglandulary tissue [8] and a thin pseudocapsule also can be seen. The typical hamartoma appearance can not be identified in breasts which are composed of dense parenchymal pattern. Additionally, the more fibroglandular tissue a hamartom contains, the more mammographically it appears to be dense; which can cause the hamartoma to interfere with the fibroadenoma [9]. Helvie et al. revealed that 30 percent of these solid lesions can not be diagnosed on MG, and there is not a typical mammographic appearance so that only 12 percent of hamartomas can be easily diagnosed without needing further evaluation [10]. On most of our cases, especially in breasts with dense parenchima patterns, mammographically appeared as heterogeneously increased asymmetric density without pseudocapsule rather than typical breast in-breast appearance or salami-slice. The contribution of ultrasonography can be restricted when an atypical appearance is encountered [11]. Our US findings were nonspecific, it was in the form of areas with heterogeneous echo that were not clearly distinguishable from the surrounding parenchyma (Figure 2).

In the setting of small lesion composed of low fat tissue and the pseudocapsule is not present reaching the accurate diagnosis can be challenging [9]. In such a small number of hamartomas with atypical appearance that borders of lesion can not be clearly identified, pathology has been resulted as normal breast tissue, so this may lead to discordance between ultrasonographic, mammographic appearance and pathologic diagnosis. The inconsistency between pathologic data and radiological data does not reassure clinicians and radiologists, which can lead to unnecessary surgical procedures. Mizuta et al. reported a case of myoid hamartoma of which could be diagnosed surgically since there was a discordance between the findings on imaging modalities and the histolopathological findings of core needle biopsy [12]. Presence of these challenges and limitations may lead clinicians and radiologists

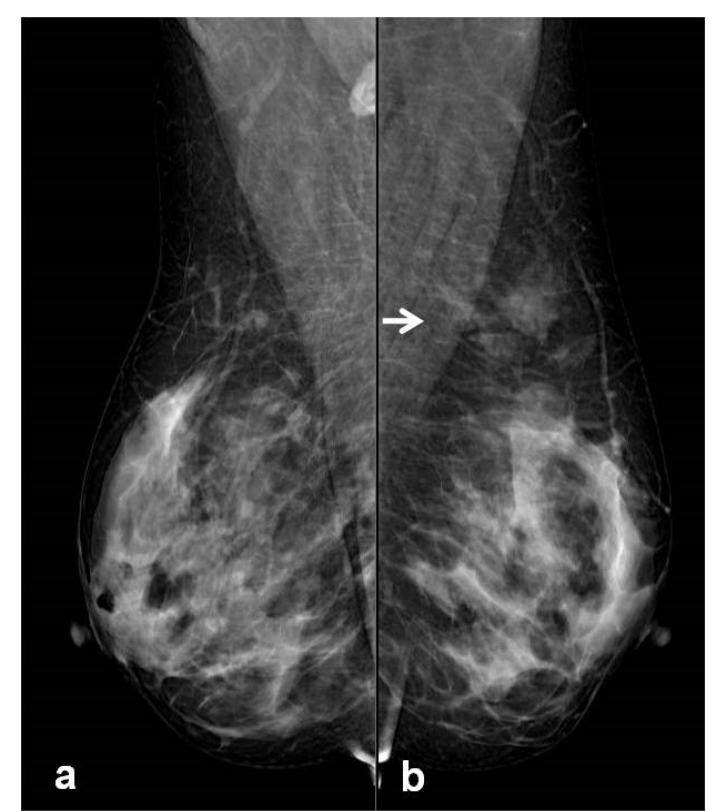

Figure 1a. On MG, image of right breast obtained from MLO. 1b. MLO imaging has demonstrated asymmetric opacity of radiolucent and dense areas; it is not distinctly encapsulated in upper outer quadrant of left breast (arrow) 
Table 1. Comparison of MRI and MG detection status of hamartom pseudo-capsule

\begin{tabular}{|c|c|c|c|c|c|c|c|}
\hline & & \multicolumn{2}{|c|}{ HPK } & \multirow[b]{2}{*}{ Total } & \multirow[b]{2}{*}{ Variable } & \multirow[b]{2}{*}{ Ratio $\pm S D$} & \multirow[b]{2}{*}{$p$} \\
\hline & & No & yes & & & & \\
\hline \multirow{3}{*}{$\begin{array}{l}\text { Pseudo-capsule of } \\
\text { Hamartoma (HPK) }\end{array}$} & Yes & 1 & 0 & 1 & $\begin{array}{l}\text { HPK that can be } \\
\text { detected by MRI }\end{array}$ & $0.964 \pm 0.188$ & \multirow{2}{*}{$<0.001$} \\
\hline & No & 17 & 10 & 27 & $\begin{array}{l}\text { HPK detected with } \\
\text { MG }\end{array}$ & $0.357 \pm 0.487$ & \\
\hline & Total & 18 & 10 & 28 & & & \\
\hline
\end{tabular}

Pseudo-capsule of Hamartoma (HPK)

Table 2. Comparison of ADC values obtained from hamartoma and normal breast tissue

\begin{tabular}{|c|c|c|c|c|c|c|c|c|}
\hline ADC & $n$ & Mean & $S D$ & Min & Max & $1 Q$ & Med & $3 Q$ \\
\hline Hamartoma & 27 & 1.44 & 0.26 & 0.8 & 2 & 1.3 & 1.5 \\
\hline $\begin{array}{c}\text { Normal breast } \\
\text { tissue }\end{array}$ & 27 & 1.43 & 0.22 & 1 & 1.9 & 1.6 & 1.3 & 1.5 \\
\hline
\end{tabular}

to need new problem solving modalities particularly in some difficult cases. Although the specificity of the dynamic-enhanced breast MRI is low compared to conventional methods, MRI is a promising method to prevent unnecessary biopsies in these difficult cases who have heterogeneous appearance, in which the lesion boundaries can not be clearly distinguished [13]. Kievit et colleagues.has reported that MRI can be facilitated reaching the accurate diagnosis by integrating the MRI to the conventional methods in selected cases in which management strategy is undetermined because of the ineffectiveness of USG and MG [14].

We presume that MRI can provide useful information to diagnose a hamartoma and prevent unnecessary biopsies in patients who do not have mammographically typical hamartoma appearance. We could easily observe the pseudo-capsule independently of parenchymal pattern; which is typical of all other patients except one patient on MRI (Figure 3a,b,c).

On our present study, enhancement patterns were also consistent with breast parenchyma or benign lesion enhancement. Furthermore, we have not detected substantial diffusion restriction on the DWI in the great majority of lesions (Figure 4a,b).

The enhancement pattern of these few lesions with mild diffusion restriction was similar to the breast parenchyma. On dynamic contrastenhanced MRI, type 1 kinetic curve is mostly seen as enhancement pattern in our patients. Erdem et colleagues revealed the contribution of not being depictured diffusion restriction of mass and being demonstrated normal ADC values of lesion in addition to USG and MG [15].

Our study has limitation: despite the high number of hamartomas evaluated, the number of patients we compared was limited since each patient was not examined with MG or MRI.

MRI can be guided by providing better understanding of the border features of the lesion comparison to MG in this group of patients, who are reported as breasts with dense paranchyma or of which is not encircled by distinct pseudocapsule. Typical pseudo-capsule of lesion and demonstrating contrast enhancement similar to breast parenchyma was useful in facilitating our diagnosis of hamartoma. Since the typical appearance of hamartoma is not seen especially in breast with dense parenchyma, it can be considered as an alternative imaging for accurate diagnosis. Conclusionally, we assume that MRI can provide more detailed information in difficult cases which have not classical mammographic appearances, so MRI can be considered as an alternative imaging for accurate diagnosis and prevent unnecessary biopsies and surgeries.

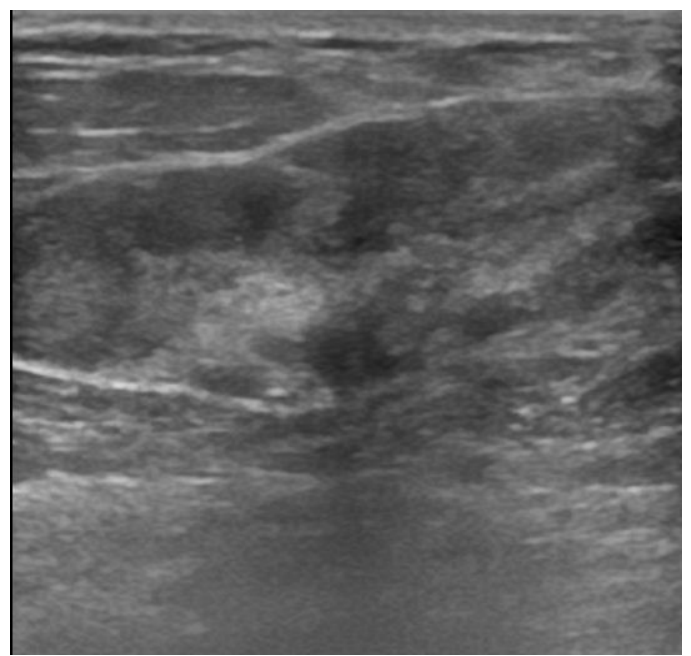

Figure 2. On USG image, heterogeneous echogenic hamartoma and pseudo-capsule is seen as thin echogenic linear lines

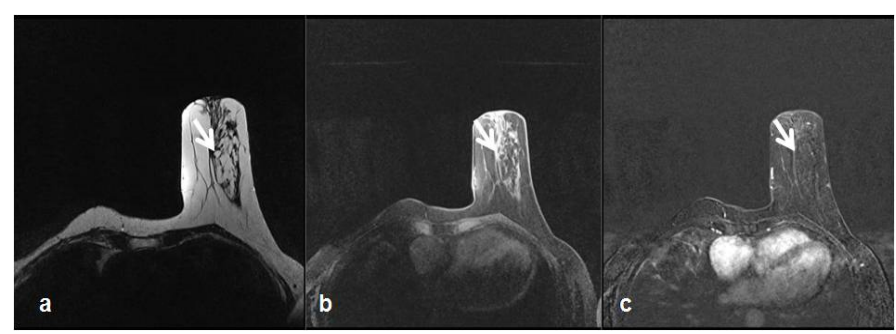

Figure 2. On USG image, heterogeneous echogenic hamartoma and pseudo-capsule is seen as thin echogenic linear lines

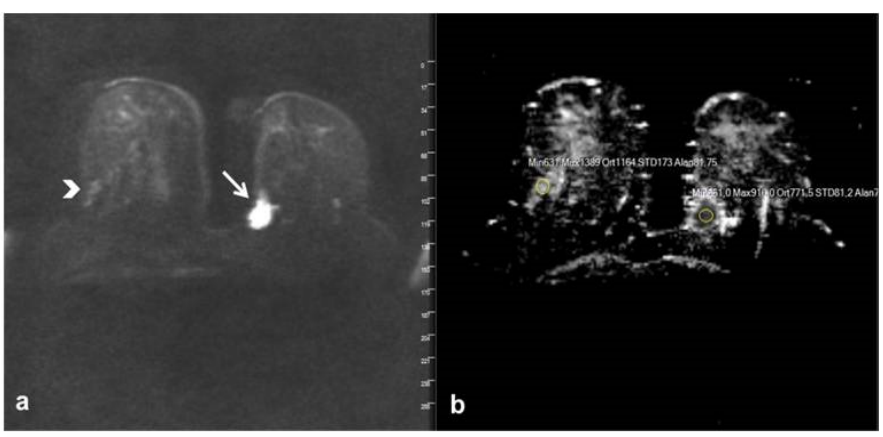

Figure 4a. On axial DWI and 4b. ADC mapping. There is no diffusion restriction seen on hamartoma with high ADC values $(>1,1)$ (Arrowhead). A mass lesion of intraductal carcinoma with a low ADC value of 0.77 showing substantial diffusion restriction in the left breast is observed (Arrow) 


\section{References}

1. Adrada B, Wu Y, Yang W (2013) Hyperechoic lesions of the breast: radiologichistopathologic correlation. AJR Am J Roentgenol 200: W518-W530. [Crossref]

2. Rohini A, Prachi K, Bhargavi V (2014) Multimodality imaging of giant breast hamartoma with pathological correlation. International J of Basic and Appl Med Sci 4: $278-281$.

3. Park SY, Oh KK, Kim EK, Son EJ, Chung WH (2003) Sonographic findings of breast hamartoma: emphasis on compressibility. Yonsei Med J 44: 847-854. [Crossref]

4. Wong KW, Ho WC, Wong TT (2002) MRI of muscular hamartoma of the breast. Australas Radiol 46: 441-443. [Crossref]

5. Harman M, Ünal Ö, Ugras S, Etlik Ö, Kotan Ç (2003) Breast hamartoma: Radiologic appearances. Eastern Journal of Medicine 8: 43-45.

6. American College of Radiology (2013) Breast imaging reporting and datasystem (BIRADS). 5th ed. Reston, Va: American College of Radiology.

7. Tatar C, Erozgen F, Tuzun S, Karsidag T, Yilmaz E, et al. (2013) Surgical approach to breast hamartoma and diagnostic accuracy in preoperative biopsies. $J$ Breast Health 9: 186-190.
8. Chao TC, Chao HH, Chen MF (2007) Sonographic features of breast hamartomas. $J$ Ultrasound Med 26: 447-452. [Crossref]

9. Presazzi A, Di Giulio G, Calliada F (2015) Breast hamartoma: ultrasound, elastosonographic, and mammographic features. Mini pictorial essay. J Ultrasound 18 : 373-377. [Crossref]

10. Helvie MA, Adler DD, Rebner M, Oberman HA (1989) Breast hamartomas: variable mammographic appearance. Radiology 170: 417-421.

11. Sanal HT, Ersoz N, Altinel O, Unal E, Can C (2006) Giant hamartoma of the breast. Breast $J$ 12: 84-85. [Crossref]

12. Mizuta N, Sakaguchi K, Mizuta M, Imai A, Nakatsukasa K, et al. (2012) Myoid hamartoma of the breast that proved difficult to diagnose: a case report. World J Surg Oncol 10: 12. [Crossref]

13. Altay C, Balci P, Altay S, Karasu S, Saydam S, et al. (2014) Diffusion-weighted MR imaging: role in the differential diagnosis of breast lesions. JBR-BTR 97: 211-216. [Crossref]

14. Kievit HCE, Sikkenk AC, Thelissen GRP, Merchant TE (1993) Magnetic resonance image appearance of hamartoma of the breast. Magnetic resonance imaging 11: 293-298.

15. Erdem G, Karakaş HM, Işı B, Frrat AK (2011) Advanced MRI findings in patients with breast hamartomas. Diagn Interv Radiol 17: 33-37. [Crossref]

Copyright: $\odot 2018$ Altunkeser A. This is an open-access article distributed under the terms of the Creative Commons Attribution License, which permits unrestricted use, distribution, and reproduction in any medium, provided the original author and source are credited. 